\title{
Measurements of the ratio of the Frank constants for splay and bend in nematics consisting of disc-like molecules
}

\section{$2,3,6,7,10,11$-hexakis ( $p$-alkoxybenzoyloxy)triphenylenes}

\author{
by T. W. WARMERDAM $\dagger$, D. FRENKEL $\ddagger$ and R. J. J. ZIJLSTRA $\dagger$ \\ $\dagger$ Fysisch laboratorium, Rijksuniversiteit Utrecht, P.O. BOX 80.000, \\ 3508 TA Utrecht, The Netherlands \\ ¥FOM Institute for Atomic and Molecular Physics, P.O. BOX 41.883, \\ 1009 DB Amsterdam, The Netherlands
}

(Received 2 September 1987; accepted 18 November 1987)

\begin{abstract}
Measurements are reported of the magnetic Frederiks transition in nematics consisting of disc-like molecules; 2, 3,6, 7, 10,11-hexakis ( $p$-alkoxybenzoyloxy)triphenylenes. In agreement with theoretical predictions [1] and previous measurements on $2,3,7,8,12,13$-hexa(alkanoyloxy)truxenes [2], we find that the splay elastic constant, $K_{1}$, is larger than the bend elastic constant, $K_{3}$. The ratio $K_{1} / K_{3}$ is remarkably constant throughout the nematic regime.
\end{abstract}

\section{Introduction}

Recently we published a paper on the measurements of the magnetic Frederiks transition in the nematic region of 2, 3, 7, 8, 12, 13-hexa(alkanoyloxy)truxenes [2]. We found that the splay elastic constant, $K_{1}$, is greater than the bend elastic constant, $K_{3}$, throughout the nematic regime, which is in agreement with a mean field theory due to Sokalski and Ruijgrok [1]. We report in the present paper the results of measurements of the magnetic Frederiks transition in the nematic region of three hexabenzoates of triphenylene; viz. 2, 3,6,7,10,11-hexakis ( $p$-hexyloxybenzoyloxy)triphenylene (1), $2,3,6,7,10,11$-hexakis ( $p$-heptyloxybenzoyloxy)triphenylene (2) and 2, 3, 6, 7, 10, 11hexakis( $p$-undecyloxybenzoyloxy)triphenylene (3) (see figure 1). These materials show a normal sequence in the occurrence of different mesophases. When cooling the isotropic liquid of these triphenylene derivatives, nematic, columnar and crystalline phases are obtained (see figure 1), in contrast to the alkanoyloxytruxenes which exhibit an inverted nematic phase, viz. a nematic phase at a lower temperature than a columnar phase $[3,4]$. In order to investigate whether the results found for the inverted nematic phase, exhibited by the truxene derivatives, are characteristic for the behaviour of nematics consisting of discoid molecules we have studied the normal nematic phase exhibited by these triphenylene derivatives.

Distortion of a uniformly aligned nematic liquid crystal raises the free energy density, which is given by (see e.g. [5])

$$
f_{\mathrm{d}}=1 / 2\left[K_{1}(\boldsymbol{\nabla} \cdot \mathbf{n})^{2}+K_{2}(\mathbf{n} \cdot \boldsymbol{\nabla} \times \mathbf{n})^{2}+K_{3}(\mathbf{n} \times \boldsymbol{\nabla} \times \mathbf{n})^{2}\right],
$$

where $f_{\mathrm{d}}$ is the distortion free energy per unit volume, and ' $K_{1}, K_{2}$ and $K_{3}$ are the curvature elastic constants, usually referred to as Frank constants, associated with splay, twist and bend distortions, respectively. The local axis of uniaxial symmetry is indicated by the director, $\mathbf{n}$, which, in discoid materials, is normal to the molecular 
<smiles>[R]c1cc2c3cc([R])c([R])cc3c3cc([R])c([R])cc3c2cc1[R]</smiles>

$\mathrm{R}$
1<smiles>[R]OC(=O)c1ccc(OCCCC)cc1</smiles>

2<smiles></smiles>

3<smiles>[R]OC(=O)c1ccc(OCCC)cc1</smiles>

$\left.1 \mathrm{~K} \stackrel{174(8.1)}{\longrightarrow}\right|_{172(1.6)} ^{\stackrel{186(2.8)}{\longrightarrow}} \mathrm{N}_{\mathrm{D}} \underset{269}{\stackrel{272(0.5)}{\longrightarrow}} \mathrm{I}$

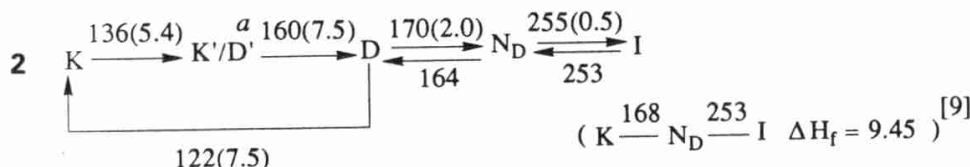

$3 \mathrm{~K} \stackrel{91}{\longrightarrow} \mathrm{K}^{\prime} / \mathrm{D}^{\prime \prime} \underset{82(4.9)}{\stackrel{125}{\rightleftarrows}} \mathrm{D}^{\stackrel{172(0.1)}{\longrightarrow}} \stackrel{191(4.3)}{\longrightarrow} \mathrm{N}_{\mathrm{D}} \stackrel{199(0.1)}{\rightleftarrows} \mathrm{I}$

$$
\left(\mathrm{K} \stackrel{145}{-} \mathrm{D}_{\mathrm{r}} \stackrel{179}{=} \mathrm{N}_{\mathrm{D}} \stackrel{185}{=} \mathrm{I} \quad \Delta \mathrm{H}_{\mathrm{f}}=25.2\right)^{[9]}
$$

Figure 1. Structures, transition temperatures and enthalpies of transitions for the triphenylene derivatives studied here. 1, 2, 3, 6, 7, 10,11-hexakis ( $p$-hexyloxybenzoyloxy)triphenylene; 2, 2, 3, 6, 7, 10, 11-hexakis( $p$-heptyloxybenzoyloxy)triphenylene; 3, 2, 3, 6, 7, 10, 11hexakis ( $p$-undecyloxybenzoyloxy)triphenylene. The transition temperature is given in ${ }^{\circ} \mathrm{C}$ and the enthalpy of the transition is indicated, between parentheses, in $\mathrm{kJ} / \mathrm{mol} . K, K^{\prime}$ indicate crystalline phases, $N_{\mathrm{D}}$ the nematic phase consisting of discoid molecules, $D, D^{\prime}$, $D_{t}, D_{r}$ columnar phases and $I$ the isotropic liquid phase. (a) Either a crystalline or a columnar phase.

plane. In the presence of a magnetic field, $\mathbf{B}$, the expression for the free energy density of a nematic material becomes

$$
f_{\text {total }}=f_{\mathrm{d}}-1 / 2 \mu_{0}^{-1} \Delta \chi(\mathbf{B} \cdot \mathbf{n})^{2}
$$

where $\Delta \chi$ is the anisotropic part of the magnetic susceptibility. Applying a magnetic field parallel to the director of a thin layer of uniformly aligned nematic material results in a deformation of the director pattern when the magnetic field strength exceeds a well-defined threshold field (the magnetic Frederiks transition) (see e.g. [6]). Studying the magnetic Frederiks transition with homeotropically aligned nematics yields information on the ratio $K_{3} / \Delta \chi$ (from the threshold field) and the ratio $K_{1} / K_{3}$ (from measurements of the degree of deformation as a function of the applied magnetic field B). 


\section{Synthesis}

The hexakis ( $p$-alkoxybenzoyloxy)triphenylenes were synthesized by the route shown in figure 2. Using the method reported by Musgrave and Webster [7] 1,2dimethoxybenzene is oxidized with $p$-chloranil in 70 per cent sulphuric acid to $2,3,6,7,10,11$-hexamethoxytriphenylene, which is subsequently demethylated in refluxing pyridine hydrochloric acid [8]. The resulting hexahydroxy compound is esterified with the appropriate carboxylic acid chloride to yield 1, 2 and $\mathbf{3}$ (see figure 1) [9]. The para-substituted alkoxybenzoic acid chlorides were synthesized following the method reported by Neubert et al. [10]. The methyl ester of $p$-hydroxybenzoic acid was synthesized by methylation of this acid [11]. The purity of the compounds was checked by N.M.R. and thin layer chromatography.

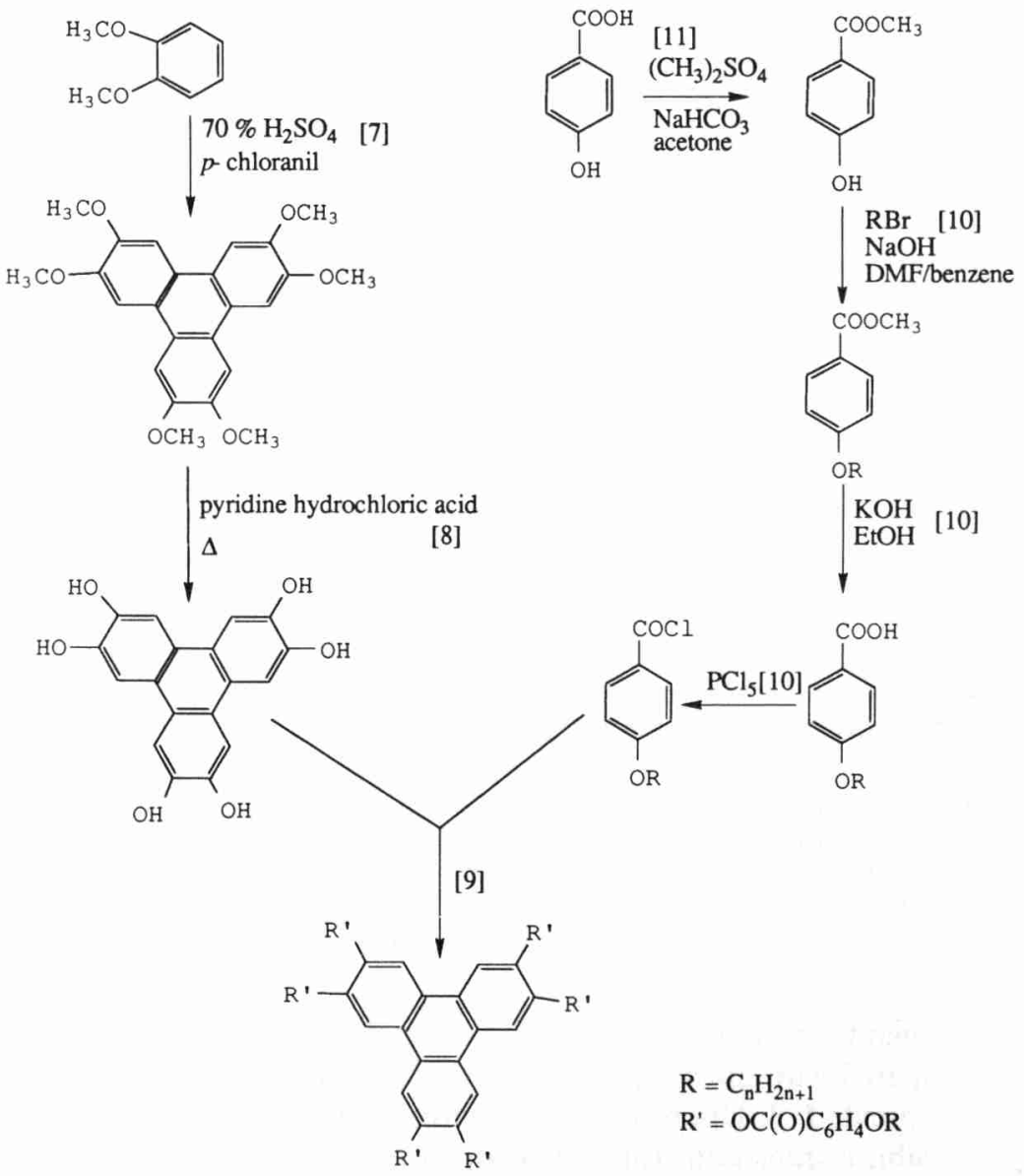

Figure 2. Synthesis of 2,3,6,7,10,11-hexakis( $p$-alkoxybenzoyloxy)triphenylenes.

\section{Optical and thermodynamic studies}

Phase transition temperatures and optical textures were studied using a polarizing microscope equipped with a Mettler FP52 heating and cooling stage. For the hexyloxy and heptyloxy derivatives $\mathbf{1}$ and $\mathbf{2}$ we found that the nematic-isotropic transition temperatures are in good agreement with literature data, whereas for the undecyloxy 

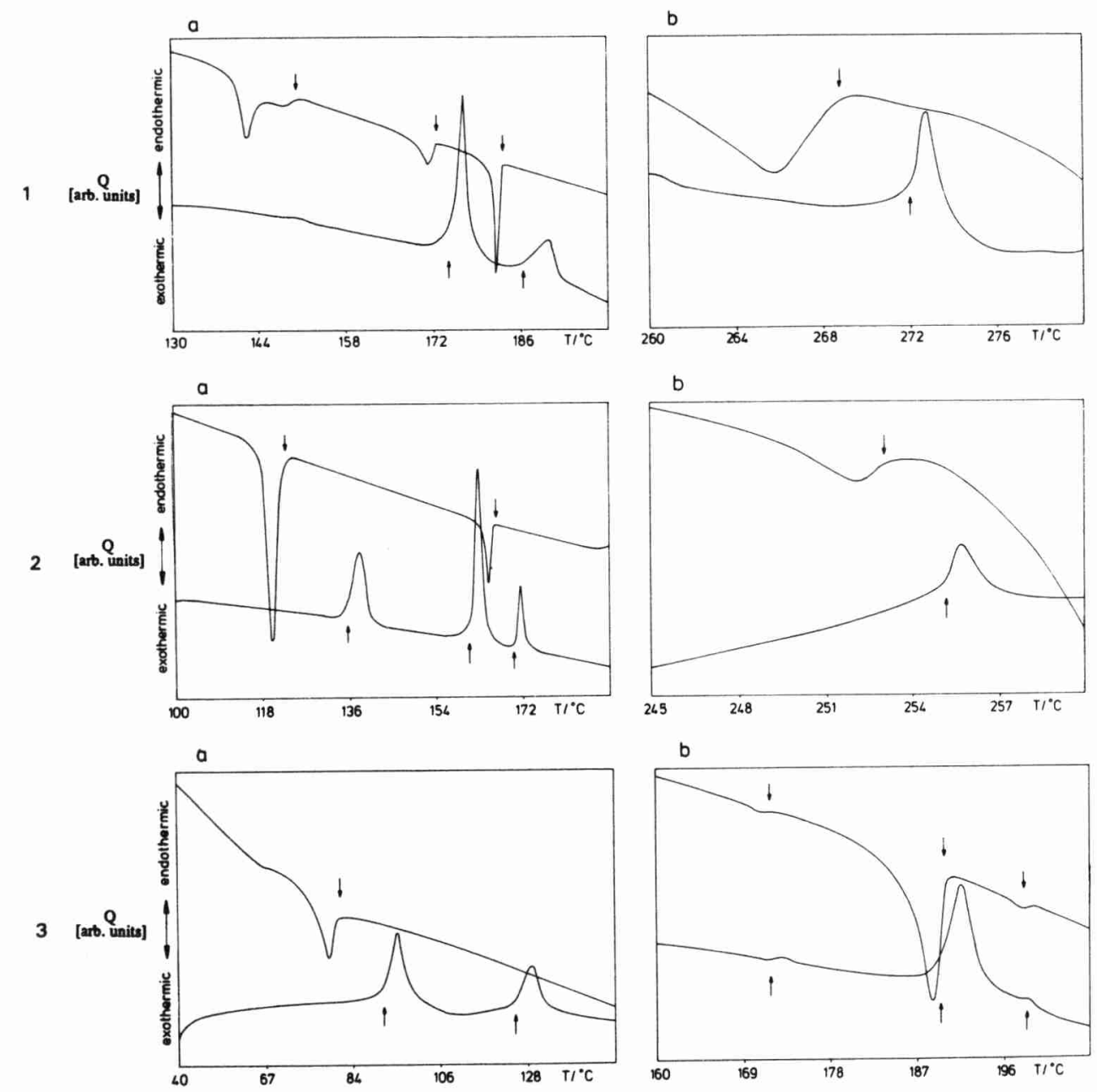

Figure 3. D.S.C. thermograms of the triphenylene derivatives 1-3. 1, 2, 3, 6, 7, 10, 11-hexakis( $p$-hexyloxybenzoyloxy)triphenylene; 2, 2,3,6,7,10,11-hexakis ( $p$-heptyloxybenzoyloxy)triphenylene; $3,2,3,6,7,10,11$-hexakis ( $p$-undecyloxybenzoyloxy)triphenylene. $Q$ is the differential heat flow. The scanning speed used was $2 \mathrm{~K} / \mathrm{min}$. The transition temperatures, as indicated by the arrows, and the corresponding enthalpies are presented in figure 1.

derivative 3 a significantly higher value was found [9]. These similar or higher clearing points as compared with the literature is another indication of the purity of the synthesized compounds 1-3. Upon cooling, all three triphenylene derivatives studied in this paper exhibit a transition from a nematic to a columnar phase. This transition was not reported in [9] for compound 2. Upon further cooling at least one more columnar phase is detected with compounds $\mathbf{1}$ and $\mathbf{3}$, which, again, to our knowledge has not been reported previously [9].

Studying these triphenylene derivatives by differential scanning calorimetry (Setaram DSC 111) confirmed the observations with the polarizing microscope (see figure 3 ). The results of the optical and thermodynamic studies are summarized in figure 1 . Behind the transition temperatures (in ${ }^{\circ} \mathrm{C}$ ), the enthalpy of the phase transition is indicated between parentheses (in $\mathrm{kJ} / \mathrm{mol}$ ). 


\section{Experimental}

Using a thin polyimide coating (Dupont, P12566) [12] and tungsten spacers as described in [2] we were able to prepare homeotropically aligned samples (viz. the director is normal to the sample walls) with a thickness of some $50 \mu \mathrm{m}$. The exact thickness of the samples was determined interferometrically. The alignment of the samples was almost perfect as far as we could tell from examination with a polarizing microscope. Using the method described in [2] a well-defined single domain magnetic deformation of the director pattern can be obtained. The amplitude of this deformation can be determined optically $[13,14]$. In the experiments the transmission of a polarized light beam through an analyser, where polarizer and analyser are mutually perpendicular and make an angle of $45^{\circ}\left(-45^{\circ}\right)$ with the plane defined by the director and the direction of the magnetic field, is monitored (see figure 4). Due to the optical anisotropy of nematic liquid crystals there are two principal refractive indices. The incident linearly polarized light wave can be decomposed into two perpendicular linearly polarized light waves, which propagate with different velocities inside the nematic material. The ordinary refractive index $\left(n_{0}\right)$ is observed for the light wave where the electric vector is perpendicular to the optical axis (viz. perpendicular to the local director $\mathbf{n}$ ), whereas the extraordinary refractive index is observed for the light wave perpendicular to this ordinary wave (viz. in the $x z$ plane defined by the director and the direction of the magnetic field). The phase difference $(\Delta \phi)$ between these two light waves after passing through a sample with thickness $d$ is given by (see e.g. [6])

$$
\Delta \phi=2 \pi d\left(n_{\mathrm{e}}-n_{\mathrm{o}}\right) / \lambda,
$$

where $\lambda$ is the wavelength of the polarized light. The ordinary refractive index is not dependent on rotation of $\mathbf{n}$ in the $x z$ plane, whereas the extraordinary refractive index depends on the rotation of $\mathbf{n}$ as given by

$$
n_{\mathrm{o}}=n_{\perp} ; \quad n_{\mathrm{e}}=n_{\|} n_{\perp} /\left(n_{\|}^{2} \cos ^{2} \theta+n_{\perp}^{2} \sin ^{2} \theta\right)^{1 / 2},
$$

where $n_{\perp}$ and $n_{\|}$are the refractive indices perpendicular and parallel to the director, respectively, and $\theta$ is the angle between the director and the direction of the magnetic field. In order to obtain information about the elastic constants we need to know the

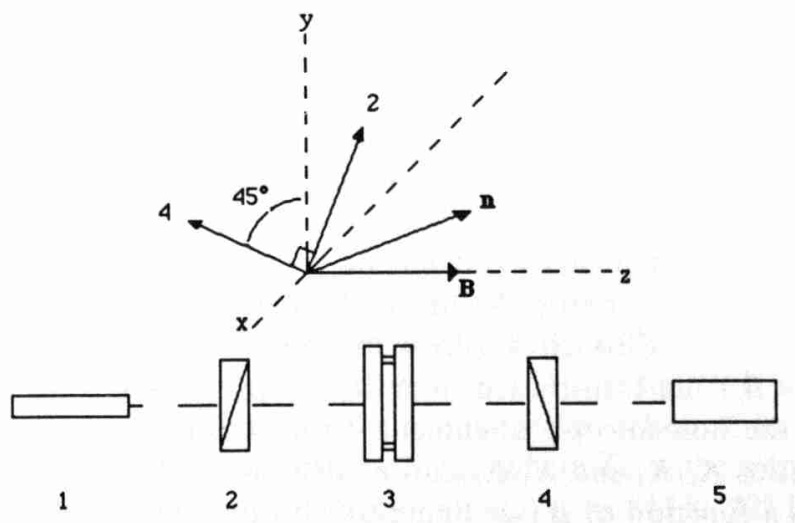

Figure 4. Experimental set-up to determine the ratios $K_{1} / K_{3}$ and $K_{3} / \Delta \chi$ in the homeotropic configuration. 1, He-Ne laser; 2 , polarizer; 3 , sample cell; 4 , analyser; 5 , photomultiplier tube. $x, y$ and $z$ indicate the axes of a right-handed cartesian coordinate system, where the $z$ axis is chosen parallel to the direction of the magnetic field. 


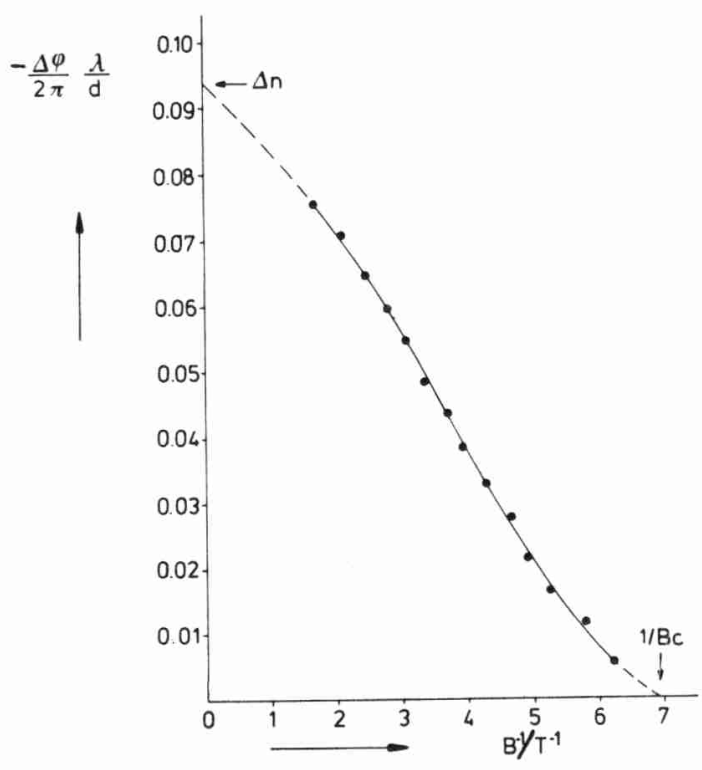

Figure 5. Typical example of a plot of $\Delta \phi \lambda / 2 \pi d$ versus $1 / B$, where $\Delta \phi$ is the phase difference between the ordinary and the extraordinary ray and $B$ is the magnetic field strength. The data shown were measured for 2, 3, 6, 7, 10,11-hexakis ( $p$-hexyloxybenzoyloxy)triphenylene 1 at $465 \mathrm{~K}$, using a sample cell of thickness $d=59 \mu \mathrm{m}$ and a $\mathrm{He}-\mathrm{Ne}$ laser with wavelength $\lambda=632.8 \mathrm{~nm}$. Extrapolation to $1 / B=0$ yields the optical anisotropy in the refractive index $(\Delta n)$. Extrapolation to $\Delta \phi=0$ yields the reciprocal value of $B_{c}$.

optical anisotropy $\Delta n=n_{\|}-n_{\perp}$. The value of $\Delta n$ will be negative because the director is perpendicular to the molecular plane. It was not possible to measure the refractive indices over the entire nematic temperature range using a temperature controlled refractometer (Zeiss, Abbe 2), because the specifications did not allow measurements at temperatures exceeding $200^{\circ} \mathrm{C}$. However, it was possible to obtain $\Delta n$ in the following way. From equation (4) it follows that $n_{\mathrm{e}}=n_{\|}$for $\theta=90^{\circ}$, which situation is reached in the limit $B=\infty$. In this limiting case $n_{\mathrm{e}}-n_{\mathrm{o}}=n_{\|}-n_{\perp}=$ $\Delta n$ and equation (3) becomes $\Delta \phi=2 \pi d \Delta n / \lambda$. The ratio $\Delta \phi \lambda / 2 \pi d$ was plotted as a function of $1 / B$. A typical example of such a plot is given in figure 5 . An estimate of $\Delta n$ was obtained from the intercept $(1 / B=0)$ of a quadratic least squares fit to the data points. These estimated values of $\Delta n$ were corrected by using them as an extra fit variable in the fitting procedure to obtain the elastic ratios $K_{1} / K_{3}$ and $K_{3} / \Delta \chi$ (vide infra) [13].

In the actual measurements the magnetic field is decreased gradually (a typical field sweep takes about 1 hour). As the field strength is varied the intensity of the transmitted light goes through a succession of minima and maxima. Below the threshold field $\left(B_{c}\right)$ the transmitted intensity drops to zero indicating a complete restoration of the homeotropic alignment. From the position of the maxima and minima, the ratios $K_{1} / K_{3}$ and $K_{3} / \Delta \chi$ can be determined by fitting the experimental curve of $\Delta \phi$ as a function of $B$ (see figure 6) with the theoretical expression [13,14] using a non-linear least squares fit. A good fit of the theoretical curve to the data points was obtained (a typical value for the sum of squares of residuals was $0 \cdot 2$ ). The estimated error in the ratio $K_{3} / \Delta \chi$ due to the fitting procedure is approximately 1 per cent. The estimated error in the ratio $K_{1} / K_{3}$ due to the fitting procedure is somewhat 


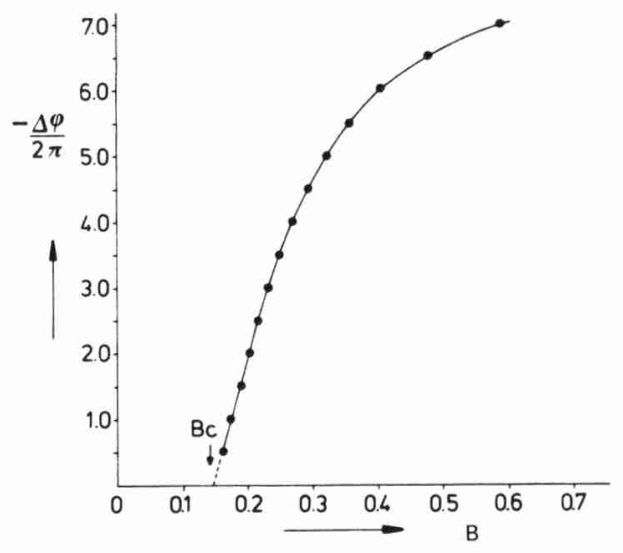

Figure 6. Typical example of $(\Delta \phi / 2 \pi)$ obtained from the minima and maxima of the optical interference pattern as a function of the magnetic field strength $(B) . \Delta \phi$ is the phase difference between the ordinary and the extraordinary ray. The data shown were measured for 2, 3, 6, 7, 10,11-hexakis( $p$-alkoxybenzoyloxy)triphenylene 1 at $465 \mathrm{~K}$, using a sample cell of thickness $d=59 \mu \mathrm{m}$ and a He-Ne laser with wavelength $\lambda=632.8 \mathrm{~nm}$. Extrapolation of the data points to $\Delta \phi=0$ yields the critical value of the magnetic field $\left(B_{c}\right)$.

larger, but does not exceed 4 per cent. Just as was observed in our previous experiments [2], however, it was found that due to the high viscosity of the triphenylene derivatives 1-3 [15] (especially at low temperatures) studied in the present experiments, the relaxation of the director distortion was very sluggish. Consequently, the positions of the maxima and minima in transmitted intensity were measured as a function of scanning speed. The positions of the maxima and minima were found to depend linearly on the scanning speed [2]. Estimates for the positions of the extrema at zero scanning speed were obtained from the intercepts of the straight lines that were fitted to the data points. These extrapolated values of the magnetic field constituted the input data used in the fitting procedure.

All measurements of the Frederiks transitions were carried out using a temperature controlled cell. In this cell the temperature could be held constant to better than $0 \cdot 1 \mathrm{~K}$, although the estimated error in the absolute temperature reading was somewhat larger. The locations of all phase transitions were checked independently by using a Mettler FP5 microscope in combination with a Mettler FP52 hot stage. In order to prevent oxidation at the high temperatures used in the present experiments all measurements were carried out under a nitrogen atmosphere.

\section{Results and discussion}

Figures 7 and 8 show the results for $\Delta n$ (figure 7) and for the ratios $K_{1} / K_{3}$ and $K_{3} / \Delta \chi$ (figure 8) of the triphenylene derivatives 1-3 (see figure 1) as a function of temperature. The corresponding numbers have been collected in the table. The temperature is expressed in reduced units $T / T_{\mathrm{Nl}}$, where $T_{\mathrm{Nl}}$ is the transition temperature from the nematic to the isotropic liquid phase. $T_{\mathrm{NI}}=545 \mathrm{~K}, 528 \mathrm{~K}$ and $472 \mathrm{~K}$ for the derivatives 1,2 and 3, respectively. The experimental results for $\Delta n$, below $200^{\circ} \mathrm{C}$, found for compound $\mathbf{2}$ agree well with data obtained with a temperature controlled Abbe refractometer (see figure 7). There is also good agreement in this low temperature region with literature data, where $\Delta n$ has been estimated from electric permittivity 


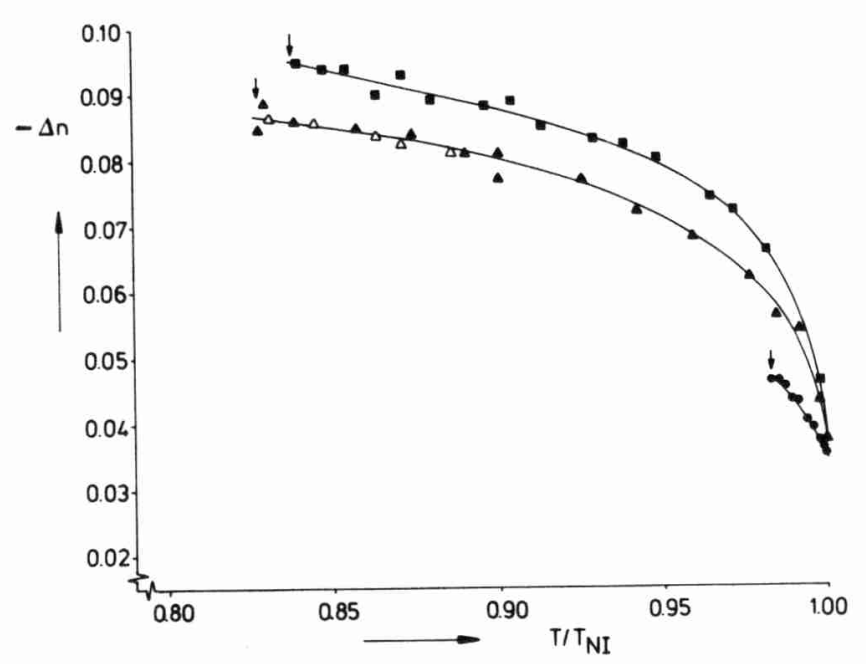

Figure 7. Temperature dependence of the optical anisotropy of the refractive index $(\Delta n)$ for the triphenylene derivatives 1-3. The estimated error in $\Delta n$ is 5 per cent. $1, T_{\mathrm{NI}}=545 \mathrm{~K}$ (closed squares); $2, T_{\mathrm{NI}}=528 \mathrm{~K}$ (closed triangles); $3, T_{\mathrm{NI}}=472 \mathrm{~K}$ (closed circles). The reduced temperature is defined as $T / T_{\mathrm{NI}}$, where $T_{\mathrm{NI}}$ is the transition temperature from the nematic to the isotropic phases. The open triangles give data points measured with a temperature controlled Abbé refractometer (Zeiss, Abbé II) for compound 2. The lower limit of the nematic regime is indicated by arrows.

measurements [16]. At $T_{\mathrm{NI}}$, however, significantly different values are obtained $(\Delta n \approx-0.03$ in the present experiments compared with $\Delta n \approx 0.00$ in [16]). A value of $\Delta n=0$ at $T_{\mathrm{NI}}$ would imply a continuous phase transition, contrary to what is commonly observed for the nematic-isotropic transition (see e.g. [6]). The occurrence of a (weakly) first order transition at $T_{\mathrm{NI}}$, as is indicated by the discontinuity of $\Delta n$ at $T_{\mathrm{NI}}$ in the present experiments, is supported by our calorimetric results (see figure 3), since they clearly indicate a value of $\Delta H \neq 0$ at $T_{\mathrm{NI}}$.

The temperature dependence of the order parameter $S$ for a wide variety of nematic rod-like liquid crystals has been studied by Buka and de Jeu [17], who measured the diamagnetic anisotropy of these materials. Assuming that the temperature dependence of $S$ can be described by the relation

$$
S=\Delta \chi^{\mathrm{m}} / \Delta \chi_{0}^{\mathrm{m}}=\left(1-y T / T_{\mathrm{NI}}\right)^{z},
$$

where $\Delta \chi_{0}^{\mathrm{m}}$ is the maximum anisotropy at $T=0$, they found values for $y$ in the range $0.9975 \pm 0.0024$ and for $z=0.18 \pm 0.07$. To our knowledge, no corresponding fits of the temperature dependence of $S$ in nematics consisting of disc-like molecules have been attempted. We tried to deduce the temperature dependence of $S$, for the materials discussed here, from the behaviour of the optical birefringence. The temperature dependence of $\Delta n$ should be approximately proportional to the nematic order parameter $S$ [6]. Similar to the relation employed by Buka and de Jeu, we used

$$
\begin{aligned}
S & =\Delta n / \Delta n(0) \\
& =\left(1-y T / T_{\mathrm{NI}}\right)^{z},
\end{aligned}
$$

where $\Delta n(0)$ is the maximum optical anisotropy at $T=0$ and can be considered to correspond to the case of a perfectly aligned nematic material. Haller's extrapolation 

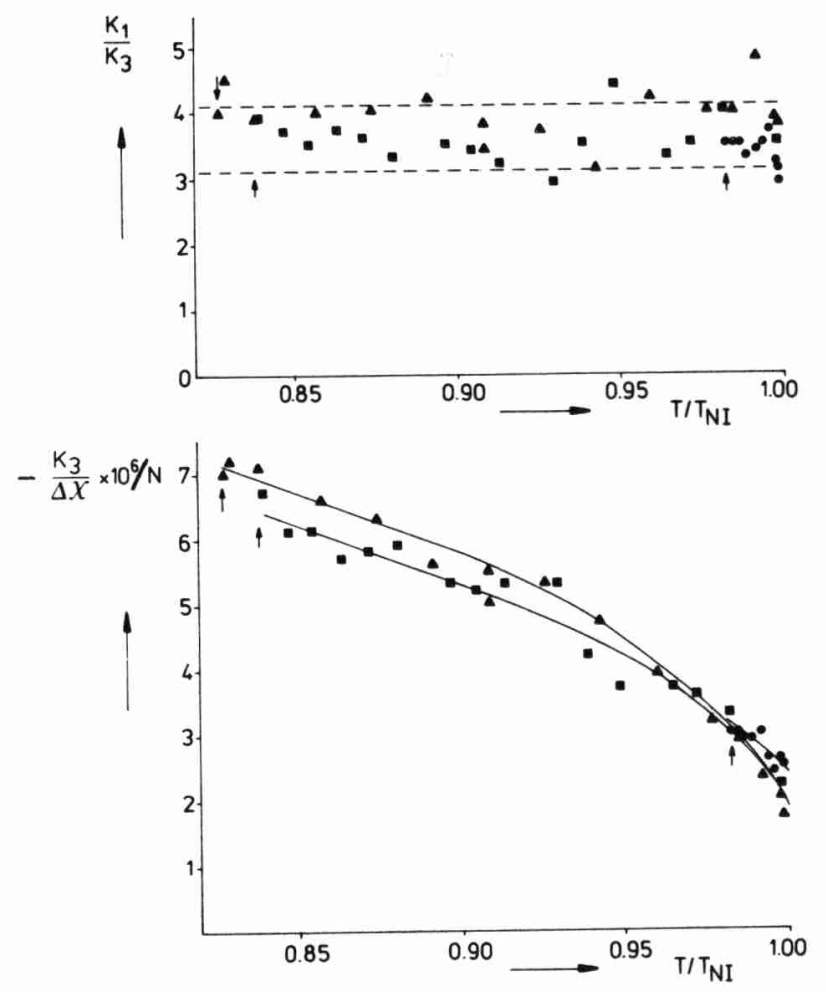

Figure 8. Temperature dependence of the ratios $K_{1} / K_{3}$ and $K_{3} / \Delta \chi$ for the triphenylene derivatives 1-3. 1, $T_{\mathrm{NI}}=545 \mathrm{~K}$ (closed squares); $2, T_{\mathrm{NI}}=528 \mathrm{~K}$ (closed triangles); 3, $T_{\mathrm{NI}}=$ $472 \mathrm{~K}$ (closed circles). The estimated error in the ratio $K_{3} / \Delta \chi$ is 10 per cent and in the ratio $K_{1} / K_{3}$ is 15 per cent. The reduced temperature is defined as $T / T_{\mathrm{NI}}$, where $T_{\mathrm{NI}}$ is the transition temperature from the nematic to the isotropic phases. The lower limit of the nematic regime is indicated by arrows.

procedure $[18,19]$ to determine the nematic order parameter from the refractive indices could not be used, because it is based on the Vuks equation [20] which requires knowledge of both $n_{\mathrm{e}}$ and $n_{\mathrm{o}}$. Since only values for $\Delta n$ could be determined in the entire nematic temperature range, the approximation in equation 5 was used. Keyes [21] has predicted that, under certain conditions, a value of $z=0.25$ is to be expected on theoretical grounds. This prediction should apply to both rod-like and disc-like molecules. In fact, there is some evidence from computer simulation studies [22] that for certain disc-like particles an exponent $z \approx 0 \cdot 25$ is indeed observed. However, until now, no experimental data on nematics consisting of disc-like molecules were available. For the three triphenylene derivatives studied values of $y \geqslant 0.997$ and $z=0.17 \pm$ 0.01 were found, clearly showing a remarkable resemblance to the data obtained for nematics consisting of rod-like molecules [17]. These results indicate that the temperature dependence of $S$ for rod-like and disc-like nematogens is indeed similar, as was expected from computer simulation studies [22]. Although the values which we obtain for $z$ appear to support the hypothesis of Keyes that the nematic-isotropic transition is tricritical-like, with a theoretical value of $z=0 \cdot 25$, rather than critical-like, which would yield $z=0.5$, not too close to the critical point [17,21], it should be noted that other predictions of Keyes' theory have not been confirmed by experiment [23]. 


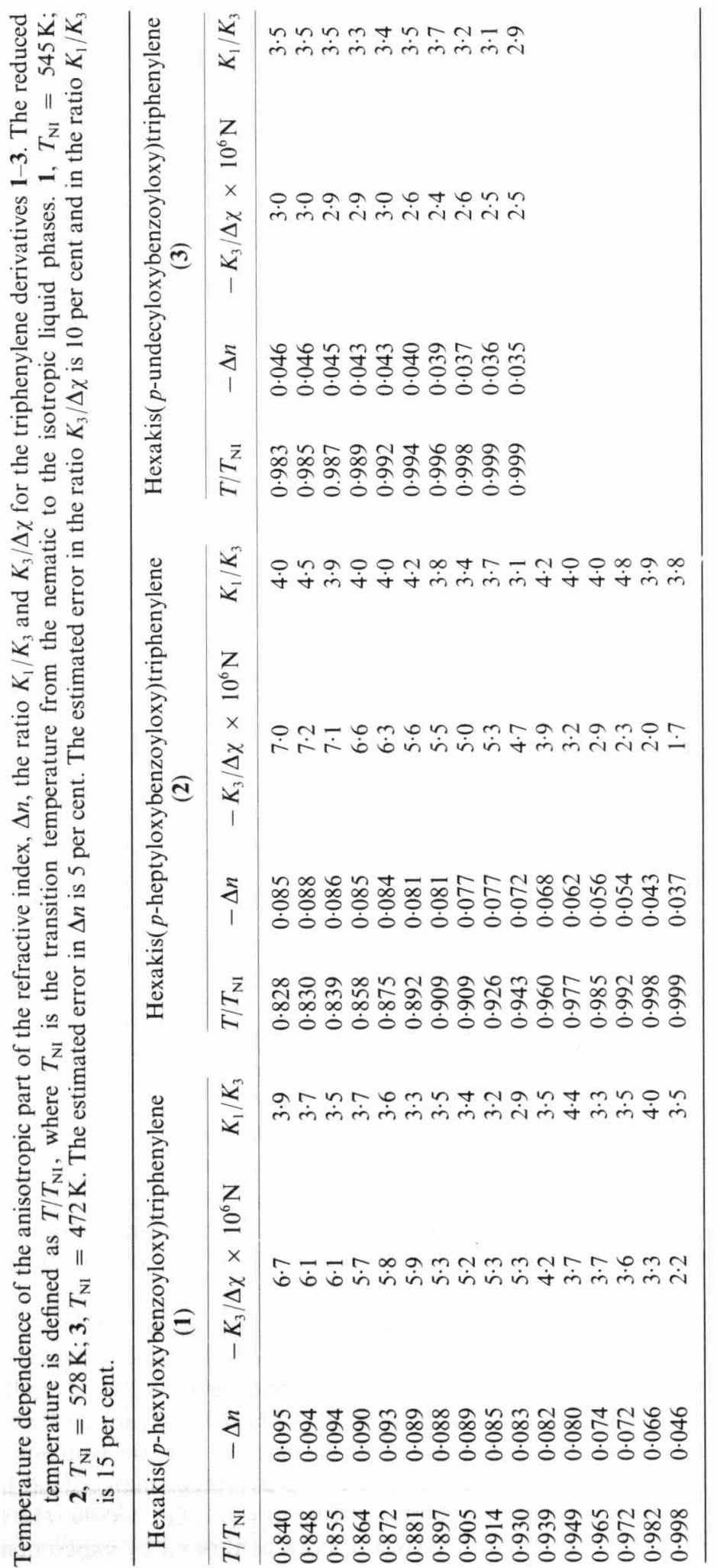


The values found for $\Delta n(0)$ decrease on increasing alkyl chain length. Values of $\Delta n(0)$ of $-0.127,-0.117$ and -0.087 were found for compounds $\mathbf{1}, \mathbf{2}$ and $\mathbf{3}$, respectively. This decrease on increasing alkyl chain length is to be expected since the optical anisotropy is mainly caused by the aromatic core [6]. On increasing alkyl chain length the volume fraction of the aromatic core decreases and so does the optical anisotropy. The value of the nematic order parameter $S$ can be calculated from the values of $\Delta n$ (in the table) and $\Delta n(0)$ (quoted previously). Typical values for $S$ vary from $0 \cdot 3-0 \cdot 4$ close to the nematic-isotropic transition to $0 \cdot 7-0 \cdot 8$ close to the nematiccolumnar transition. These values are comparable to those found for nematics which consist of rod-like molecules and which exhibit a broad nematic region $\left(>50^{\circ} \mathrm{C}\right)[6]$.

From figure 8 it is clear that the ratio $K_{1} / K_{3}$ is remarkably constant over the entire nematic temperature range for all three derivatives 1-3. Several authors $[14,24,25]$ have concluded from calculations and measurements that for nematics in the limit of small $S, K_{1}=K_{3}$ and all three elastic constants are proportional to $S^{2}$. Hence $K_{\alpha} \approx c_{\alpha}$ $S^{2}\left(\alpha=1,2\right.$ or 3 , representing splay, twist and bend, respectively), where $c_{\alpha}$ is a constant that depends only on molecular properties. Due to this proportionality the ratio $K_{1} / K_{3}$ is expected to be a constant independent of temperature. However, close to the nematic-isotropic transition the value of $S$ is still in the region $0 \cdot 3-0 \cdot 4$ which cannot be considered as being the limit of small $S$. For values of $S$ which are not small $K_{1} \neq K_{3}$ is commonly found, and the values of $K_{1}$ and $K_{3}$ approach each other with increasing temperature [6]. In the present experiments it was found also that $K_{1} \neq K_{3}$, but no systematic trend with temperature was apparent from our data. We note here that in our experiments on alkanoyloxytruxenes [2], which exhibit an inverted nematic phase [26, 27], the ratio $K_{1} / K_{3}$ decreases slightly with increasing temperature for the decanoyloxy and tetradecanoyloxy derivatives, whereas the ratio $K_{1} / K_{3}$ was almost a constant for the octadecanoyloxy derivative [15]. In this latter compound the nematic phase appears to be followed by an isotropic phase [15], similar to the triphenylene derivatives studied in the present experiments, whereas in the former compounds the nematic phase is followed by a columnar one.

In agreement with a mean field theory due to Sokalski and Ruijgrok [1] and with previous measurements on alkanoyloxytruxenes [2] the present experiments show a larger value for the splay elastic constant $K_{1}$ than for the bend elastic constant $K_{3}$. The order of magnitude of the ratio $K_{3} / \Delta \chi$ in the present experiments is also similar to the data obtained previously and is comparable to what is commonly found in nematics consisting of rod-like molecules. An estimate of the absolute values of the elastic constants can be made using the values for $\chi_{\text {isotropic }}-\chi_{\perp}\left(\Delta \chi=\chi_{\|}-\chi_{\perp}=3\left(\chi_{\text {isotropic }}-\right.\right.$ $\left.\chi_{\perp}\right)$ as reported by Levelut et al. [28]. Close to the nematic-isotropic transition temperature values of $\Delta \chi^{\mathrm{m}}$ of $-0.39 \times 10^{-7}$ and $-0.21 \times 10^{-7} \mathrm{uem} \mathrm{CGS} \mathrm{g}^{-1}$ were reported for compounds 1 and 3 respectively. Assuming a value of $0.8 \mathrm{~g} / \mathrm{cm}^{3}$ for the density and multiplying by a factor $4 \pi$ for conversion to SI units, this leads to absolute values of the bend elastic constants close to $T_{\mathrm{NI}}$ of approximately $9 \times 10^{-13} \mathrm{~N}$ and $5 \times 10^{-13} \mathrm{~N}$ for compounds 1 and 3 , respectively. This value increases on cooling and at approximately $254^{\circ} \mathrm{C}\left(T / T_{\mathrm{NI}}=0.963\right)$, the lower limit of the $\Delta \chi$ measurements reported in [28], a value of $K_{3} \approx 2 \times 10^{-12} \mathrm{~N}$ is obtained for compound 1 . The corresponding absolute values of $K_{1}$ are a factor approximately 3.6 larger. We note that measurements of the electric Frederiks transition in the nematic phase of compound 2 yield a comparable value for $K_{1}\left(K_{1}=3.5 \times 10^{-12} \mathrm{~N}\right.$ at $\left.230^{\circ} \mathrm{C}\right)$ [16].

A Landau theory proposed by Swift and Andereck [29] predicts an enhancement of the splay elastic constant $K_{1}$ due to fluctuations of a columnar order parameter on 
approaching the nematic-columnar transition temperature, whereas $K_{3}$ would not exhibit pretransitional effects. If the transition is an (almost) second order phase transformation, theory [29] predicts that the ratio $K_{1} / K_{3}$ would in fact diverge at the transition temperature. We do not find evidence, however, for such behaviour. Neither was this the case in the previously studied nematic phase of alkanoyloxytruxenes [2].

The refractive index measurements reported in this paper for compound $\mathbf{2}$ below $200^{\circ} \mathrm{C}$ were performed on the temperature controlled Abbe refractometer of the University of Groningen. We thank Mr. E. Gramsbergen and Professor W. H. de Jeu for their kind assistance in these measurements. The synthesis of the compounds described in this paper was carried out in collaboration with Dr. R. J. M. Nolte and Professor W. Drenth of the Department of Physical and Organic Chemistry of the University of Utrecht.

\section{References}

[1] Sokalski, K., and Ruijgrok, Th. W., 1982, Physica A, 113, 126.

[2] Warmerdam, T. W., Frenkel, D., and Zijlstra, R. J. J., 1987, J. Phys., Paris, 48, 319.

[3] Destrade, C., Nguyen Huu Tinh, Gasparoux, H., Malthete, J., and levelut, A. M., 1981, Molec. Crystals liq. Crystals, 71, 111.

[4] Destrade, C., Gasparoux, H., Foucher, P., Nguyen Huu Tinh, Malthete, J., and JAQUES, J., 1983, J. Chim. phys., 80, 137.

[5] De Gennes, P. G., 1974, The Physics of Liquid Crystals (Clarendon Press).

[6] De Jeu, W. H., 1980, Physical Properties of Liquid Crystalline Materials (Gordon \& Breach).

[7] Musgrave, O. C., and Webster, C. J., 1971, J. chem. Soc. C, p. 1397.

[8] Prey, V., 1941, Ber. dt. chem. Ges., 74, 1219; 1942, Ibid., 75, 350, 445, 537. Curphey, T. J., Hoffman, E. J., and McDonald, C., 1967, Chem. Ind., p. 1138.

[9] Nguyen Huu Tinh, Gasparoux, H., and Destrade, C., 1981, Molec. Crystals liq. Crystals, 68, 101.

[10] Neubert, M. E., Laskos, S. J., Jr., Maurer, L. J., Carlino, L. T., and Ferrato, J. P., 1978, Molec. Crystals liq. Crystals, 44, 197.

[11] Ramao Rao A. V., Madhusudan N. Deshmukh and latha Sivadashan, 1981, Chem. Ind., p. 164.

[12] Van Sprang, H. A., 1983, J. Phys., Paris, 44, 421.

[13] Bunning, J. D., Faber, T. E., and Sherrell, P. L., 1981, J. Phys., Paris, 42, 1175.

[14] Leenhouts, F., and Dekker, A. J., 1981, J. chem. Phys., 74, 1956.

[15] Warmerdam, T. W., Frenkel, D., and Zijlstra, R. J. J., Liq. Crystals (to be published).

[16] Mourey, B., Perbet, J. N., Hareng, M., and Le Berre, S., 1982, Molec. Crystals liq. Crystals, 84, 193.

[17] BukA, A., and de Jeu, W. H., 1982, J. Phys., Paris, 43, 361.

[18] Haller, I., 1975, Prog. solid St. Chem., 10, 103.

[19] Tough, R. J. A., and Bradshaw, M. J., 1983, J. Phys., Paris, 44, 447.

[20] Vuks, M. F., 1966, Opt. Spectrosc., 20, 644.

[21] Keyes, P. H., 1978, Physics Lett. A, 67, 132.

[22] Eppenga, R., and Frenkel, D., 1984, Molec. Phys., 52, 1303.

[23] Rosenblatt, C., 1983, Phys. Rev. A Rap. Commun., 27, 1234.

[24] Nehring, J., and Saupe, A., 1972, J. chem. Phys., 56, 5527.

[25] Priest, R. G., 1973, Phys. Rev. A, 7, 720.

[26] Nguyen Huu Tinh, Foucher, P., Destrade, C., Levelut, A. M., and Malthete, J., 1984, Molec. Crystals liq. Crystals, 111, 277.

[27] Nguyen Huu Tinh, Malthete, J. and Destrade, C., 1981, Molec. Crystals liq. Crystals, 64, 291.

[28] Levelut, A. M., Hardouin, F., Gasparoux, H., Destrade, C., and Nguyen Huu Tinh, 1981, J. Phys., Paris, $42,147$.

[29] Swift, J., and Andereck, B. S., 1982, J. Phys. Lett., Paris, 43, 437. 